\section{Biometric and refractive changes after orbital decompression in Korean patients with thyroid-associated orbitopathy}

WS Kim¹, YS Chun ${ }^{1}$, BY Cho ${ }^{2}$ and JK Lee ${ }^{1,2}$
${ }^{1}$ Department of Ophthalmology, ChungAng University College of Medicine, Chung-Ang University Hospital, Seoul, Korea

${ }^{2}$ Thyroid Center, ChungAng University College of Medicine, Chung-Ang University Hospital, Seoul, Korea

Correspondence: JK Lee, Department of Ophthalmology, ChungAng University College of Medicine, Chung-Ang University Hospital, 102 Heukseok-ro, Dongjak-gu, Seoul 156-755, Korea Tel: +82 26299 1665; Fax: +82 28251665 . E-mail: Ik1246@gmail.com

Received: 3 June 2015 Accepted in revised form: 11 October 2015 Published online: 20 November 2015

\begin{abstract}
Purpose To determine the biometric and refractive changes after orbital decompression in Korean patients with thyroid-associated orbitopathy (TAO).

Methods Retrospective, observational study (between October 2012 and September 2014) was performed. Patients with TAO undergoing orbital decompression for stable proptosis received ophthalmic examinations, including Hertel exophthalmometry, A-scan biometry, autorefraction measures, corneal topography, and wavefront aberration measures, before orbital decompression and again 2 months after surgery.

Results Included in the study were 43 eyes from 23 patients. The mean exophthalmometric value decreased by $4.1 \mathrm{~mm} 2$ months after orbital decompression $(P<0.001)$. On average, axial length $(\mathrm{AL})$ increased significantly by $0.08 \mathrm{~mm}(P<0.001)$; specifically, $37(86 \%)$ of the 43 eyes had increased AL. Whereas anterior chamber depth and lens thickness showed no significant changes $(P=0.086$ and $P=0.905$, respectively), the mean spherical refraction and spherical equivalent (SE) decreased by 0.35 and $0.48 \mathrm{D}$, respectively $(P=0.008$ and $P<0.001$, respectively). However, cylindrical refraction and axis showed no significant changes ( $P=0.057$ and $P=0.218$, respectively). The changes in AL and SE were significantly correlated $(R=-0.411, P=0.009)$. Notably, there were no changes in corneal topography or wavefront aberration after orbital decompression.

Conclusions TAO patients who underwent orbital decompression showed myopic refractive change via increase in AL. Possible
\end{abstract}

refractive changes should be considered in cases of TAO complaining of decreased visual acuity after orbital decompression. Eye (2016) 30, 400-405; doi:10.1038/eye.2015.242; published online 20 November 2015

\section{Introduction}

The prevalence of thyroid-associated ophthalmopathy (TAO) is $>30 \%$ in Asian and Caucasian patients with Graves' disease, ${ }^{1}$ and TAO is one of the most common cause of orbital inflammatory disorders in adults. Pathological changes in TAO are related to $\mathrm{T}$ and $\mathrm{B}$ lymphocytes, along with various cytokines and chemokines. Longstanding inflammation induces adipogenesis, hyaluronic acid deposition, secondary hydration, and finally, ocular muscle thickening and proliferation of fatty tissue. ${ }^{2}$ Proliferated fatty tissue and engorged muscles occupy the retrobulbar space and push the eyeball forward, often resulting in proptosis. Complications due to eyeball compression, such as increased intraocular pressure, primary open angle glaucoma, ${ }^{3,4}$ and optic neuropathy, ${ }^{5}$ are clinically significant problems.

As proptosis progresses, the four rectus muscles, the medial, superior, inferior, and lateral rectus muscles, elongate. The contractile force of the extraocular muscles (EOMs) is positively correlated to the EOM length. ${ }^{6,7}$ Proliferative retrobulbar tissue pushes the eyeball forward and elongates the four rectus muscles, causing them to pull the eyeball more strongly posterior. Therefore, the direction of the final vector on the eyeball results in a compressive force parallel to the visual axis, increasing the amplitude of the vector. 
This mechanism may induce changes in ocular biometrics and alter the refractive error. However, in TAO patients, the correlations between proptosis progression, biometric changes, and refractive error have not yet been investigated.

Orbital decompression is a useful procedure to alleviate proptosis in TAO patients. For patients undergoing successful decompression, we can estimate ophthalmic status prior to the start of proptosis. Although there have been studies reporting the effect of orbital decompression on refraction, ${ }^{8-10}$ whether or not refractive error is induced or not remains uncertain. In this study, we assessed the biometrics of the eyeball, including axial length (AL), anterior chamber depth (ACD), lens thickness (LT), refraction, keratometry, and aberration, before and after orbital decompression in order to estimate the correlation between proptosis progression and biometric changes.

\section{Materials and methods}

We completed a retrospective chart review of patients undergoing orbital decompression for stable proptosis secondary to TAO from 7 October 2012 to 21 September 2014. This study was approved by the Chung-Ang University Hospital Institutional Review Board and adhered to the tenets of Declaration of Helsinki.

The diagnosis of TAO was based on orbital computed tomography scan findings of EOM enlargement or muscle proliferation, in addition to serological results, a history of endocrine findings, and one or more clinical findings, including upper eyelid retraction, proptosis, and EOM restriction. We performed orbital decompression if patients' clinical activity score was $<3$ out of 10 , that is, inflammation was inactive, and exophthalmometry was stable. Criteria for exclusion were past ocular surgery, grade $\geq 2$ corneal erosion (Oxford scheme ${ }^{11}$ ), cataract, vitreous opacity, retinal disorders, and other diseases affecting the refractive error or AL of the eyeball.

Before orbital decompression and again 2 months after decompression, all patients underwent ophthalmic examinations, including Hertel Exophthalmometry, A-scan Biometry (Aviso, Quantel Medical, Cournon d'Auvergne Cedex, France) including AL, LT, and ACD, autorefraction (KR-8800, Topcon, Tokyo, Japan), corneal topography (ATLAS, Carl Zeiss Meditec, Oberkochen, Germany), and wavefront aberration (WASCA Analyzer, Carl Zeiss Meditec). Orbital decompression was performed by a single surgeon (JKL), and balanced decompression (deep lateral, medial wall decompression) or three-wall decompression (deep lateral, medial, inferior walls) with or without fat excision was selected based on the degree of proptosis.
Preoperative measurements of AL, LT, and ACD were obtained via contact ultrasonic A-scan by a single examiner (WSK). The cornea was topically anesthetized with one drop of $0.5 \%$ proparacaine hydrochloride (Alcaine, Alcon, Fort worth, Texas, USA). Each eye was measured 10 times using a laser-guided probe to ensure proper alignment perpendicular to the visual axis. An echogram was accepted if all four echoes, including those of the corneal surface, anterior and posterior lens surfaces, and the retina, were as perpendicular and steep as possible and also if the SD from 10 measurements was $<50 \mu \mathrm{m}$.

Autorefraction was performed three times by a single examiner (WSK). If the three results did not agree, then the test was repeated. Spherical refraction (Dsph), cylindrical refraction (Dcyl), cylindrical axis, and spherical equivalent (SE) were recorded.

Wavefront aberration was measured by a single examiner (WSK) using a WASCA Analyzer under photopic conditions $\left(85 \mathrm{~cd} / \mathrm{m}^{2}\right)$.

Analytic comparisons between preoperative and postoperative data from the A-scans, autorefractor, topography, and aberrometer were performed using paired T-tests. Correlations between exophthalmometry, $\mathrm{AL}$, and refractive changes were evaluated using the Pearson correlation test. A level of $P<0.05$ was accepted as statistically significant. Data were analyzed using SPSS (Version 20, IBM, Armonk, NY, USA).

\section{Results}

Forty-three eyes from 23 patients were included over the study period. Mean age was $30.5 \pm 8.9$ years, and the male-to-female ratio was 7 (14 eyes):16 (29 eyes). The mean exophthalmometric value changed significantly by $-4.1 \mathrm{~mm} 2$ months after decompression, ranging from 3.0 to $5.0 \mathrm{~mm}(P<0.001$; Table 1$)$. Among the total 43 eyes, myopia, emmetropia, and hyperopia was observed in 34 (79.0\%), 7 (16.3\%), and 2 (4.7\%) eyes, respectively.

Table 1 Participant demographics

\begin{tabular}{|c|c|c|}
\hline Total subjects & 23 & \\
\hline Total eyes & 43 & \\
\hline $\mathrm{M}: \mathrm{F}$ & 7 (14 eyes):16 (29 eyes) & \\
\hline Mean age, years & $30.5 \pm 8.9(17-52)^{\mathrm{a}}$ & \\
\hline \multicolumn{3}{|l|}{ Mean Exo. (mm) } \\
\hline Pre-Op & $19.6 \pm 2.4(17-25)^{\mathrm{a}}$ & $P<0.001^{\mathrm{b}}$ \\
\hline Post-Op & $15.5 \pm 2.1(13-20)^{\mathrm{a}}$ & \\
\hline
\end{tabular}

Abbreviation: Exo., exophthalmometry.

${ }^{\text {a }}$ Mean \pm SD (range).

${ }^{\mathrm{b}}$ Paired $T$-test. 


\section{Eyeball biometrics}

Two months after surgery, the mean AL increased significantly by $0.08 \mathrm{~mm}(P<0.001)$. However, ACD and LT showed no significant changes $(P=0.086$ and $P=0.905$, respectively; Table 2$)$. Only $5(12 \%)$ of the 43 eyes had decreased $A L$, and $1(2 \%)$ subject had the same AL before and after orbit decompression. Notably, 37 eyes (86\%) had increased AL after decompression. However, ACD increased in 27 (63\%) eyes and decreased in 16 (37\%) eyes. LT increased in $18(42 \%)$ eyes and decreased in $22(51 \%)$ eyes, and $3(7 \%)$ eyes showed no change (Figure 1a).

\section{Refractive error}

Before and 2 months after orbital decompression, Dsph and SE changed significantly $(P=0.008$ and $P<0.001$, respectively). Specifically, the mean Dsph and SE decreased by 0.35 and $0.48 \mathrm{D}$, from -3.37 to $-3.72 \mathrm{D}$ and from -3.92 to $-4.40 \mathrm{D}$, respectively. However, Dcyl and cylindrical axis measures were not significantly changed $(P=0.057$ and $P=0.218$, respectively; Table 2$)$.

Table 2 Biometric and refractive changes of the eyeball before and 2 months after orbital decompression

\begin{tabular}{|c|c|c|}
\hline & Value $^{\mathrm{a}}$ & P-value ${ }^{\mathrm{b}}$ \\
\hline \multicolumn{3}{|l|}{$A L(\mathrm{~mm})$} \\
\hline Pre-op & $24.82 \pm 1.18$ & \multirow[t]{2}{*}{$<0.001$} \\
\hline Post-op & $24.90 \pm 1.18$ & \\
\hline \multicolumn{3}{|l|}{$A C D(\mathrm{~mm})$} \\
\hline Pre-op & $3.52 \pm 0.38$ & \multirow[t]{2}{*}{0.086} \\
\hline Post-op & $3.57 \pm 0.34$ & \\
\hline \multicolumn{3}{|l|}{$L T(m m)$} \\
\hline Pre-op & $3.82 \pm 0.30$ & \multirow[t]{2}{*}{0.905} \\
\hline Post-op & $3.82 \pm 0.32$ & \\
\hline \multicolumn{3}{|l|}{ Dsph (D) } \\
\hline Pre-op & $-3.37 \pm 3.71$ & \multirow[t]{2}{*}{0.008} \\
\hline Post-op & $-3.72 \pm 3.52$ & \\
\hline \multicolumn{3}{|l|}{$\operatorname{Dcyl}(D)$} \\
\hline Pre-op & $-1.10 \pm 0.79$ & \multirow[t]{2}{*}{0.057} \\
\hline Post-op & $-1.32 \pm 0.63$ & \\
\hline \multicolumn{3}{|c|}{ Cylindrical axis $\left(^{\circ}\right)$} \\
\hline Pre-op & $176 \pm 15$ & \multirow[t]{2}{*}{0.218} \\
\hline Post-op & $186 \pm 15$ & \\
\hline \multicolumn{3}{|l|}{$S E(D)$} \\
\hline Pre-op & $-3.92 \pm 3.72$ & \multirow[t]{2}{*}{$<0.001$} \\
\hline Post-op & $-4.40 \pm 3.63$ & \\
\hline
\end{tabular}

Abbreviations: $\mathrm{ACD}$, anterior chamber depth; $\mathrm{AL}$, axial length of eyeball; Dcyl, cylindrical refraction; Dsph, spherical refraction; LT, lens thickness; $\mathrm{SE}$, spherical equivalent.

${ }^{\mathrm{a}}$ Mean \pm SD.

${ }^{\mathrm{b}}$ Paired T-test.
Correlations between exophthalmometry, $A L$, and refractive error

Differences in exophthalmometry and AL 2 months after orbital decompression were positively correlated.

However, this correlation was not statistically significant (Pearson correlation coefficient, $R=0.329, P=0.118$;

Figure $1 b)$. Although there was no significant correlation between the differences in AL and spherical error (Pearson correlation coefficient, $R=-0.263, P=0.073$; Figure 1c), the change in SE was significantly correlated to AL (Pearson correlation coefficient, $R=-0.411, P=0.009$; Figure 1d). Specifically, as the AL increased, the myopic shift was increased.

\section{Corneal topogram}

Although the mean simulated $K$ decreased by $0.25 \mathrm{D}$, this change was not significant $(P=0.759)$. The corneal irregularity measurement (CIM), which is a variable indicating asphericity, showed no significant changes after decompression $(P=0.113$ and $P=0186$ respectively; Table 3).

\section{Wavefront aberration}

The root mean square of total aberration and high-order aberration (HOA) showed no significant changes after orbital decompression $(P=0.437$ and $P=0.553$, respectively; Table 3). Also, the change in HOA, Zernike polynomials from $Z(2,-2)$ to $Z(4,4)$ was not significant.

\section{Conclusion}

In this study, we investigated the changes in eyeball biometrics, refractive error, corneal topography, and wavefront aberration before and 2 months after orbital decompression in TAO patients. The AL of the eyeball increased, and Dsph and SE were found to be myopicshifted. Notably, the changes in AL and SE were significantly correlated, such that as AL increased after orbital decompression, the myopic shift also increased. However, biometrics, including LT, ACD, corneal topographic value, and aberration, including total and high order aberrations, showed no changes after orbital decompression.

As TAO progresses, EOM thickening and fat proliferation in the retrobulbar space are generally aggravated, while the bony orbit surrounding the orbital space maintains a consistent volume. Thus increases in intra-orbital pressure occur, and the eyeball is pushed outward from the orbit. Simultaneously, a reactive force is required to hold the eyeball in a state of dynamic equilibrium. That is, the tensile force of the four rectus 

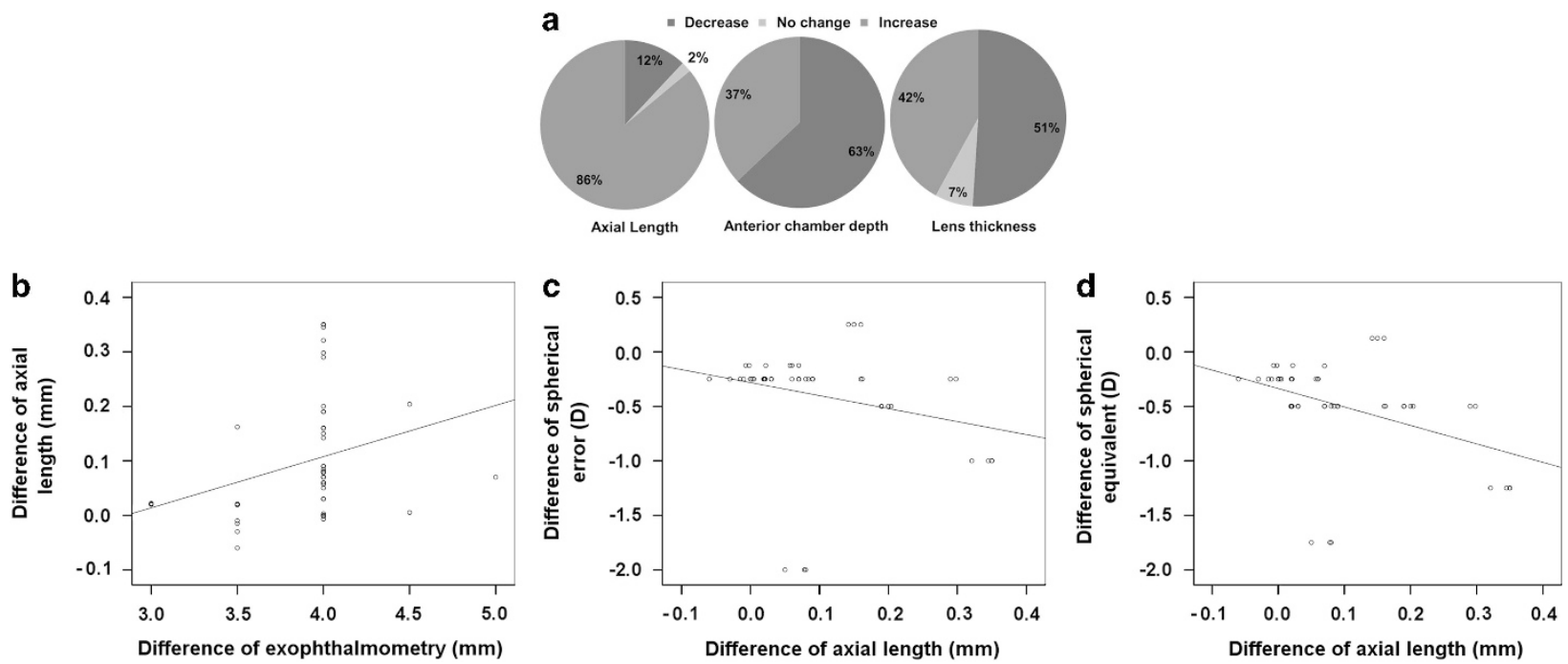

Figure 1 Proportion of biometrical changes and graphs showing the correlation between differences in exophthalmometry, AL, and refractive error before and 2 months after orbital decompression. (a) Only five (12\%) of 43 eyes had decreased AL, and one subject (2\%) had the same AL before and after orbit decompression. However, 37 cases (86\%) had increased AL. ACD increased in 27 eyes (63\%) and decreased in 16 eyes (37\%). LT increased in 18 eyes (42\%), decreased in 22 eyes $(51 \%)$, and showed no change in three eyes $(7 \%)$. (b) Differences in exophthalmometry and AL before and after orbital decompression were positively correlated. However, this correlation was not statistically significant (Pearson correlation coefficient, $R=0.329, P=0.118$ ). (c) Although the correlation between difference in AL and spherical error was not significant (Pearson correlation coefficient, $R=-0.263, P=0.073$ ), SE was significantly correlated to AL. (d) As AL increased, SE decreased (Pearson correlation coefficient, $R=-0.411, P=0.009$ ).

Table 3 Topographic and aberrational changes before and 2 months after orbital decompression

\begin{tabular}{lcc}
\hline & Value $^{\mathrm{a}}$ & P-value $^{\mathrm{b}}$ \\
\hline K (D) & & \\
Pre-op & $42.27 \pm 5.05$ & 0.759 \\
Post-op & $42.02 \pm 5.72$ & \\
& & \\
CIM (D) & & 0.113 \\
Pre-op & $1.35 \pm 1.01$ & \\
Post-op & $1.15 \pm 0.58$ & \\
& & \\
Shape factor (D) & & \\
Pre-op & $0.23 \pm 0.47$ & \\
Post-op & $0.33 \pm 0.87$ & \\
& & \\
Total aberration (RMS) & & \\
Pre-op & $3.84 \pm 3.30$ & \\
Post-op & $4.00 \pm 3.20$ & \\
& & \\
High-order aberration (RMS) & & \\
Pre-op & $0.55 \pm 0.20$ & \\
Post-op & $0.57 \pm 0.21$ &
\end{tabular}

Abbreviations: CIM, corneal irregularity measurement; RMS, root mean square.

${ }^{\mathrm{a}}$ Mean \pm SD.

${ }^{b}$ Paired $T$-test.

muscles, which include the medial, lateral, superior, and inferior rectus muscles, may increase. One previous study that indirectly supports our hypothesis reported two cases of rectus muscle palsy with a passive ocular proptosis of $4 \mathrm{~mm}^{4}$ Increased exophthalmos would elongate the rectus muscles, and because the length and force of the EOM are positively correlated, ${ }^{6,7}$ the backward force of the EOM on the eyeball increases (Figure 2a).

In a stable state of proptosis, the increased forces that push the posterior pole and pull the insertion site of the four rectus muscles are balanced. These two forces may induce a compressive change to the elastic eyeball. One case series has reported flattening of the posterior pole in MRI findings of a TAO patient. ${ }^{10}$

After orbital decompression, the retro-orbital space is enlarged in volume as the bony structures are removed. This reduces retrobulbar pressure, resolves exophthalmos, and finally, may reverse the increased AL of the elastic eyeball (Figure $2 b$ ). In our study, the mean AL was significantly increased by $0.08 \mathrm{~mm}$ after surgery compared with before surgery. Only $14 \%$ of 43 eyes had decreased or no change in AL after surgery, whereas $86 \%$ had an increased AL.

There have been a few studies reporting the effect of orbital decompression on refraction. ${ }^{8-10}$ However, whether or not myopic shift is induced by surgery is uncertain. In our study, the mean spherical refraction was decreased by $0.38 \mathrm{D}$ with the myopic change. One previous study explored the correlation between refractive error and orbital tumors occupying the retrobulbar space and reported that hypermetropic change is induced by the tumor and that tumor removal normalizes the hypermetropic change. ${ }^{13,14}$ According to 


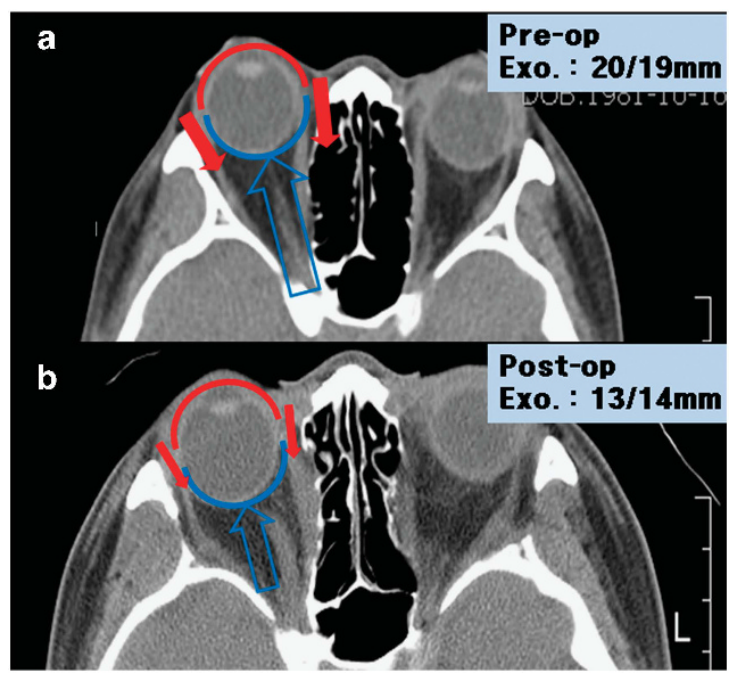

Figure 2 Computed tomographic images showing the orbital changes before and after orbital decompression. (a) EOM thickening and fat proliferation increase intra-orbital pressure and push the eyeball outward from the orbit. Simultaneously, reactive forces are needed to keep the eyeball in a state of equilibrium. That is, tensile forces of the four rectus muscles pull the eyeball backward. (b) Two months after orbital decompression, the retro-orbital volume increased. This reduced the retrobulbar pressure, resolved the exophthalmos, and reversed the change in AL of the elastic eyeball. Pre-op, pre-operation; Post-op, post-operation; Exo., exophthalmometry.

the SRK-2 formula, ${ }^{15}$ a $+0.078-\mathrm{mm}$ increase in ocular AL is equal to an approximate $0.2-\mathrm{D}$ decrease. In this study, we verified the refractive change after orbital decompression. We found the mean spherical refractive change to be $-0.35 \mathrm{D}$, which is similar to the calculated value from the ARK-2 formula. Additionally, elongation of the AL was significantly correlated to SE changes (Figure 1d).

Therefore, we concluded that myopic change was induced by AL elongation. However, in contrast to the presence of tumors, which induce astigmatism, ${ }^{13,16}$ there were no significant changes in cylindrical refraction or axis in our study (Table 2), because orbital decompression symmetrically widened the space (Figure 2).

We found no significant correlation between AL and exophthalmometric change (Figure 1b). Ocular rigidity, which is related to the pressure-volume curve, is different for each individual. ${ }^{17}$ In addition, exophthalmometry was measured at $0.5-\mathrm{mm}$ intervals, and differences in exophthalmometry ranged from 3 to $5 \mathrm{~mm}$. Therefore, many participants had same values, especially $4 \mathrm{~mm}$ (Figure 1b). To precisely evaluate the correlation between changes in exophthalmometry and AL, more detailed examination is needed.

In addition, keratometric data from the topogram, which included simulated K, CIM, and shape factor, also did not change significantly after decompression. As proptosis was relieved, rectus muscle tension decreased. However, these changes may have influenced the posterior portion from the muscle insertion site. Moreover, longstanding proptosis could result in permanent corneal shape changes. ${ }^{13}$ Accordingly, corneal shape might not be affected by orbital decompression. In addition, results from the wavefront aberrometer showed that postsurgical changes in highorder aberration were not significant (Table 3). If the forces produced a complex change in the contour of the ocular surface, then the corneal shape and aberration would also have changed. ${ }^{16,18}$

Our study has some limitations. First, although autorefractive keratometry is a precise and reproducible tool, ${ }^{19}$ it is not the gold standard for evaluating refractive error. ${ }^{20,21}$ To combat this issue, we repeated our examination three times, and if the three results did not agree with each other, then the test was repeated. In addition, the examination was performed by a single examiner (WSK) using the same device before and after surgery. Second, 43 eyes is a small population. However, it is notable that only $14 \%$ of the eyes had decrease or no change in AL, and $86 \%$ of all cases had showed an increase in AL after surgery. Third, $79.0 \%$ of the total eyes were myopia, and because the scleral rigidity of myopic eyes is weaker than hypermetropic and emmetropic eyes, ${ }^{22}$ our results including AL elongation and myopic shift might be affected by high proportion of myopia. Finally, we performed postoperative examinations 2 months after surgery, that is, our follow-up period is short. However, Chang et al ${ }^{23}$ reported that it took only about 2 months to show the maximal effect of bony decompression.

In conclusion, TAO patients who undergo orbital decompression will experience a myopic refractive change via increase in AL. On the other hand, keratometry and aberration do not change after orbital decompression. We conclude that hypermetropic change can be induced by the progression of TAO. Thus, if TAO patients complain of decreased visual acuity, physicians should rule out the progression of TAO and possible subsequent refractive change.

\section{Summary}

\section{What was known before}

- Orbital decompression is a useful procedure to alleviate proptosis in TAO patients.

- There have been studies reporting the effect of orbital decompression on refraction, whether or not refractive error is induced or not remains uncertain.

What this study adds

- TAO patients who underwent orbital decompression showed myopic refractive change via increase in AL.

- There were no changes in corneal topography or wavefront aberration after orbital decompression. 


\section{Conflict of interest}

The authors declare no conflict of interest.

\section{References}

1 Lim SL, Lim AK, Mumtaz M, Hussein E, Wan Bebakar WM, Khir AS. Prevalence, risk factors, and clinical features of thyroid-associated ophthalmopathy in multiethnic Malaysian patients with Graves' disease. Thyroid 2008; 18: 1297-1301.

2 Hatton MP, Rubin PA. The pathophysiology of thyroidassociated ophthalmopathy. Ophthalmol Clin North Am 2002; 15: 113-119.

3 Cockerham KP, Pal C, Jani B, Wolter A, Kennerdell JS. The prevalence and implications of ocular hypertension and glaucoma in thyroid-associated orbitopathy. Ophthalmology 1997; 104: 914-917.

4 Ohtsuka K, Nakamura Y. Open-angle glaucoma associated with Graves disease. Am J Ophthalmol 2000; 129: 613-617.

5 Trobe JD. Optic nerve involvement in dysthyroidism. Ophthalmology 1981; 88: 488-492.

6 Robinson DA, O'Meara DM, Scott AB, Collins CC. Mechanical components of human eye movements. J Appl Physiol 1969; 26: 548-553.

7 Simonsz HJ, Kolling GH, van Dijk B, Kaufmann H. Lengthtension curves of human eye muscles during succinylcholine-induced contraction. Invest Ophthalmol Vis Sci 1988; 29: 1320-1330.

8 Sagili S, Desousa JL, Malhotra R. Intraocular pressure and refractive changes following orbital decompression with intraconal fat excision. Open Ophthalmol J 2008; 2: 73-76.

9 Norris JH, Ross JJ, Kazim M, Selva D, Malhotra R. The effect of orbital decompression surgery on refraction and intraocular pressure in patients with thyroid orbitopathy. Eye (Lond) 2012; 26: 535-543.

10 Chandrasekaran S, Petsoglou C, Billson FA, Selva D, Ghabrial R. Refractive change in thyroid eye disease (a neglected clinical sign). Br J Ophthalmol 2006; 90: 307-309.
11 Bron AJ, Evans VE, Smith JA. Grading of corneal and conjunctival staining in the context of other dry eye tests. Cornea 2003; 22: 640-650.

12 O'Neill BP. Passive ocular proptosis. J Neurol Neurosurg Psychiatry 1977; 40: 1198-1202.

13 Singh D, Pushker N, Bajaj MS, Saxena R, Sharma S, Ghose S. Visual function alterations in orbital tumors and factors predicting visual outcome after surgery. Eye (Lond) 2012; 26: 448-453.

14 Friberg TR, Grove AS. Choroidal folds and refractive errors associated with orbital tumors. Arch Ophthalmol 1983; 101: 598-603.

15 Dang MS, Raj PP. SRK II formula in the calculation of intraocular lens power. Br I Ophthalmol 1989; 73: 823-826.

16 Robb RM. Refractive errors associated with hemangiomas of the eyelids and orbit in infancy. Am J Ophthalmol 1977; 83: 52-58.

17 Silver DM, Geyer O. Pressure-volume relation for the living human eye. Curr Eye Res 2000; 20: 115-120.

18 Bessho K, Bartsch DU, Gomez L, Cheng L, Koh HJ, Freeman WR. Ocular wavefront aberrations in patients with macular diseases. Retina 2009; 29: 1356-1363.

19 Pesudovs K, Weisinger HS. A comparison of autorefractor performance. Optom Vis Sci 2004; 81: 554-558.

20 Jorge J, Queirós A, Almeida JB, Parafita MA. Retinoscopy/ autorefraction: which is the best starting point for a noncycloplegic refraction? Optom Vis Sci 2005; 82: 64-68.

21 Choong YF, Chen AH, Goh PP. A comparison of autorefraction and subjective refraction with and without cycloplegia in primary school children. Am J Ophthalmol 2006; 142: 68-74.

22 Sergienko NM, Shargorogska I. The scleral rigidity of eyes with different refractions. Graefes Arch Clin Exp Ophthalmol 2012; 250: 1009-1012.

23 Chang M, Baek S, Lee TS. Long-term outcomes of unilateral orbital fat decompression for thyroid eye disease. Graefes Arch Clin Exp Ophthalmol 2013; 251: 935-939. 